\title{
Design of A New Three Phase Hybrid H-bridge and H-Type FCMLI for Various PWM Strategies
}

\author{
C. R. Balamurugan*, S. P. Natarajan**, V. Padmathilagam***, T. S. Anandhi** \\ * Departement of Electrical and Electronics Engineering, Arunai Engineering College, India \\ ** Departement of Electronis \& Instrumentation Engineering, Annamalai University, India \\ *** Department of EEE, Annamalai University, India
}

\begin{tabular}{l}
\hline Article Info \\
\hline Article history: \\
Received Jun 18, 2015 \\
Revised Aug 12, 2015 \\
Accepted Aug 27, 2015 \\
\hline
\end{tabular}

\section{Keyword:}

FCMLI

H-Bridge

H-Type

Hybrid

THD

\begin{abstract}
The inverters have to be designed to obtain a quality output voltage or a current waveform with a minimum amount of ripple content. In high power and high voltage applications the conventional two level inverters, however, have some limitations in operating at high frequency mainly due to switching losses and constraints of the power device ratings. Series and parallel combination of power switches in order to achieve the power handling voltages and currents. The conventional two level inverters produce THD levels around $60 \%$ even under normal operating conditions which are undesirable and cause more losses and other power quality problems too on the AC drives and utilities. Nowadays, multilevel inverters are widely used in power industry. Voltage unbalance problem is one of the major issues in working of multilevel inverter. In this paper, a three phase $\mathrm{H}$-bridge $+\mathrm{H}$-type FCMLI using sinusoidal reference, third harmonic injection reference, 60 degree reference and stepped wave reference are initially developed using SIMULINK and then implemented in real time environment using dSPACE. The five level output voltages of the chosen MLIs obtained using the MATLAB and DSPACE based PWM strategies and the corresponding \% THD, $\mathrm{V}_{\mathrm{RMS}}$ (fundamental), $\mathrm{CF}$ and $\mathrm{FF}$ are presented and analyzed. It is observed that bipolar COPWM-C provides output with relatively low distortion for sine reference and bipolar COPWM-B strategy is found to perform better since it provides relatively higher fundamental RMS output voltage for 60 degree reference.
\end{abstract}

Copyright $\odot 2015$ Institute of Advanced Engineering and Science. All rights reserved.

\section{Corresponding Author:}

C. R. Balamurugan,

Departement of Electrical and Electronics Engineering,

Arunai Engineering College,

Tiruvannamalai, India.

Email: crbalain2010@gmail.com

\section{INTRODUCTION}

In the recent past, the multilevel inverters have drawn tremendous attention in the field of high voltage and high power applications. One of the most important problems in controlling a multilevel voltage source inverter is to obtain a variable amplitude and frequency sinusoidal output by employing simple control techniques. In voltage source inverters, the non fundamental current harmonics cause power losses, electromagnetic interference and pulsating torques in $\mathrm{AC}$ motor drives. So the harmonic reduction must be related to performance of an inverter. Multilevel voltage source inverter has various Pulse Width Modulation control schemes have been developed and the same were analyzed in the previous chapter with respect to reduction in power quality issues as discussed by Corzine et al (2003). Multilevel inverter can increase the power by (m-1) times than that of two level inverter through the series and parallel connection of semiconductor power switches. Compared this with two level inverter system delivers same power, the 
multilevel inverter has some advantages of that the lower harmonic components on the output AC voltages can be eliminated and EMI problem could be decreased. Babaei et al., (2014) the topology requires lesser number of power switches and dc voltage sources. Banaei et al. (2014) the proposed inverter works with lower total peak inverse voltage. Kangarlu et al., (2013) the multilevel inverter uses reduced number of power switching devices. Special focus has been paid to obtain optimal structures regarding different criteria such as number of dc voltage sources, standing voltage on the switches, number of switches. Odeh et al., (2013) analysis of the conduction power losses in the power semiconductor switches of the proposed inverter topology. Chung-Ming Young et al., (2013) the combination of batteries can be controlled according to the batteries voltages to implement the battery-balancing function. Panda et al., (2012) cascaded multilevel inverter with low-frequency three-phase transformers and a single dc power source is proposed. This topology aims to reduce the number of components and so reduce the complexity of the circuit. Zixin et al. (2012) the level of the output voltage is only half of the dc-link voltage in all conditions, leading to much reduced dv/dt. Yi-Hung et al., (2011) the studied multistring inverter topology offers strong advantages such as improved output waveforms, lower electromagnetic interference and THD. Hinago et al., (2010) the proposed inverter can output more numbers of voltage levels in the same number of switching devices by using this conversion. The number of driving circuits is reduced, which leads to the reduction of the power consumption and size in the driving circuits. The THD value of the output waveform is also reduced. Naumanem et al., (2010) pulsed inverter voltage and the impedance mismatch between the cable and the motor cause an oscillating overvoltage in the motor terminals.

\section{H-BRIDGE AND H-TYPE FCMLI}

Figure 1 shows the different types of multilevel inverters. In this work, a H-bridge will produce three output levels and H-type FCMLI will produce five output levels. Both the modules are connected in series. So the total output is $\mathrm{V}_{\mathrm{dc} 1}+\mathrm{V}_{\mathrm{dc} 2}=\mathrm{V}_{\mathrm{o}}$. The output voltage across the load will have seven levels and the number of carriers required to produce seven output levels is six carriers. Multi-carrier bipolar triangular carrier modulation strategies with different references are employed for the study of the chosen MLI. Figure 2 shows the power circuit for three phase H-bridge and H-type FCMLI. The multilevel inverters are classified as follows:

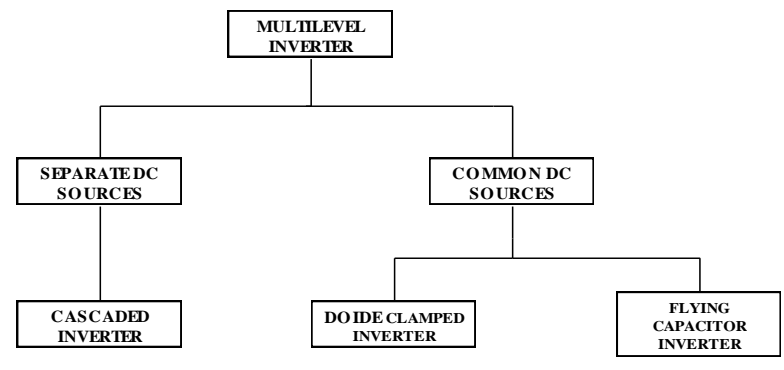

Figure 1. Types of multilevel Inverters

Table 1 displays the switch states and output voltage levels for H-bridge and H-type flying capacitor multilevel inverter.

Table 1. H-bridge and H-type flying capacitor multilevel inverter - switch states and output voltage levels

\begin{tabular}{|c|c|c|c|c|c|c|c|c|c|c|c|c|c|c|}
\hline \multirow[b]{2}{*}{$S_{\mathrm{a} 1}$} & \multicolumn{7}{|c|}{ H-type FCMLI } & \multicolumn{4}{|c|}{ H-Bridge } & \multirow[b]{2}{*}{$\mathrm{V}_{\mathrm{ao}}$} & \multirow{2}{*}{$V_{\text {bo }}$} & \multirow[b]{2}{*}{$\mathrm{V}_{\mathrm{ab}}$} \\
\hline & $\mathrm{S}_{\mathrm{a} 2}$ & $\mathrm{~S}_{\mathrm{a} 3}$ & $\mathrm{~S}_{\mathrm{a} 4}$ & $\mathrm{~S}_{\mathrm{b} 1}$ & $\mathrm{~S}_{\mathrm{b} 2}$ & $\mathrm{~S}_{\mathrm{b} 3}$ & $\mathrm{~S}_{\mathrm{b} 4}$ & $\mathrm{~S}_{\mathrm{a} 1}$ & $\mathrm{~S}_{\mathrm{a} 2}$ & $\mathrm{~S}_{\mathrm{b} 1}$ & $\mathrm{~S}_{\mathrm{b} 2}$ & & & \\
\hline 0 & 0 & 1 & 1 & 1 & 1 & 0 & 0 & 1 & 0 & 0 & 1 & $-\mathrm{Vdc}$ & Vdc & $-2 \mathrm{Vdc}$ \\
\hline 0 & 0 & 1 & 1 & 1 & 1 & 0 & 0 & 0 & 1 & 0 & 1 & $-1 / 2 \mathrm{Vdc}$ & $1 / 2 \mathrm{Vdc}$ & $-V d c$ \\
\hline 0 & 0 & 1 & 1 & 0 & 1 & 0 & 1 & 0 & 1 & 0 & 1 & $-1 / 2 \mathrm{Vdc}$ & 0 & $-1 / 2 \mathrm{Vdc}$ \\
\hline 0 & 1 & 0 & 1 & 1 & 1 & 0 & 0 & 0 & 1 & 0 & 1 & 0 & $1 / 2 \mathrm{Vdc}$ & $-1 / 2 \mathrm{Vdc}$ \\
\hline 1 & 0 & 1 & 0 & 1 & 1 & 0 & 0 & 0 & 1 & 0 & 1 & 0 & $-1 / 2 \mathrm{Vdc}$ & $1 / 2 \mathrm{Vdc}$ \\
\hline 1 & 1 & 0 & 0 & 1 & 1 & 0 & 0 & 0 & 1 & 0 & 1 & $1 / 2 \mathrm{Vdc}$ & $1 / 2 \mathrm{Vdc}$ & 0 \\
\hline 0 & 0 & 1 & 1 & 0 & 0 & 1 & 1 & 0 & 1 & 0 & 1 & $-1 / 2 \mathrm{Vdc}$ & $-1 / 2 \mathrm{Vdc}$ & 0 \\
\hline 0 & 1 & 0 & 1 & 0 & 0 & 1 & 1 & 0 & 1 & 0 & 1 & 0 & $-1 / 2 \mathrm{Vdc}$ & $-1 / 2 \mathrm{Vdc}$ \\
\hline 1 & 1 & 0 & 0 & 0 & 1 & 0 & 1 & 0 & 1 & 0 & 1 & $1 / 2 \mathrm{Vdc}$ & 0 & $1 / 2 \mathrm{Vdc}$ \\
\hline 1 & 0 & 1 & 0 & 0 & 0 & 1 & 1 & 0 & 1 & 0 & 1 & 0 & $-1 / 2 \mathrm{Vdc}$ & $1 / 2 \mathrm{Vdc}$ \\
\hline 1 & 1 & 0 & 0 & 0 & 0 & 1 & 1 & 0 & 1 & 0 & 1 & $1 / 2 \mathrm{Vdc}$ & $-1 / 2 \mathrm{Vdc}$ & Vdc \\
\hline 1 & 1 & 0 & 0 & 0 & 0 & 1 & 1 & 0 & 1 & 1 & 0 & $\mathrm{Vdc}$ & $-\mathrm{Vdc}$ & $2 \mathrm{Vdc}$ \\
\hline
\end{tabular}




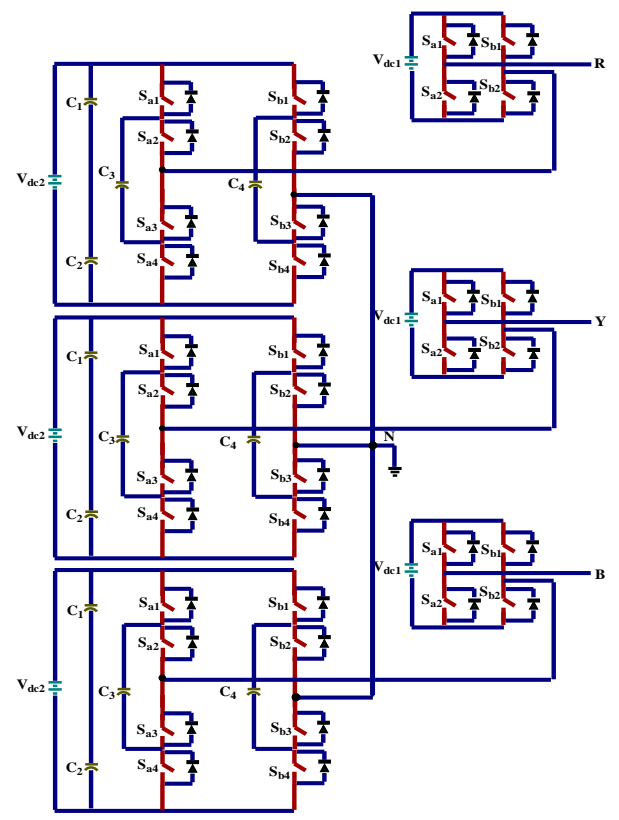

Figure 2. Three phase H-bridge and H-type flying capacitor multilevel inverter

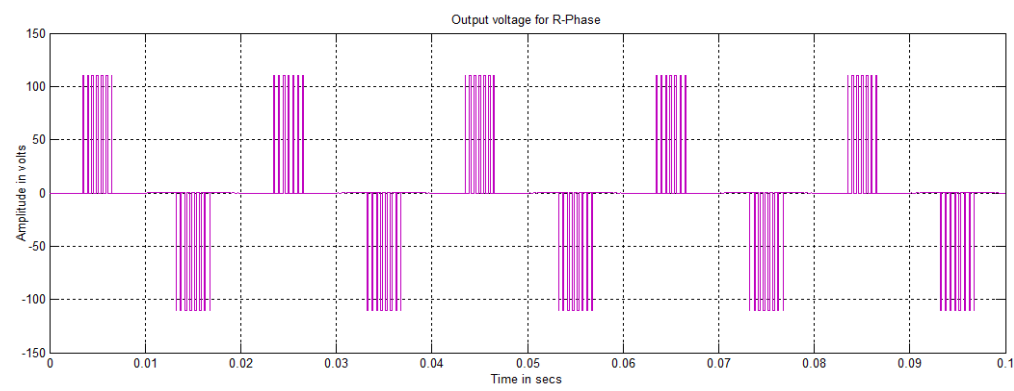

Figure 3. Output voltage of H-bridge for R-Phase

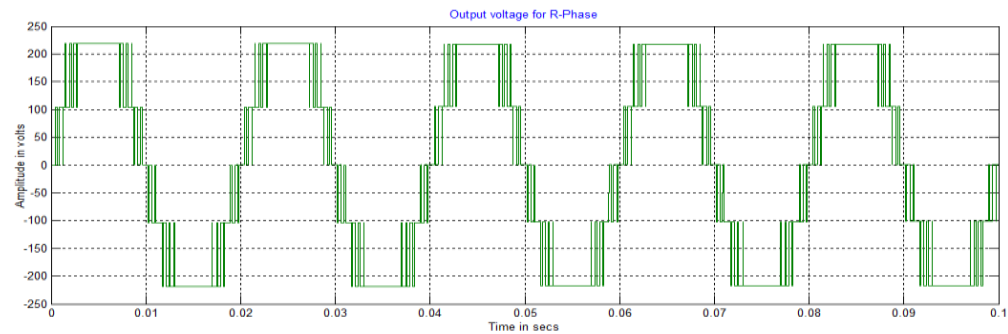

Figure 4. Output voltage of H-type FCMLI for R-Phase

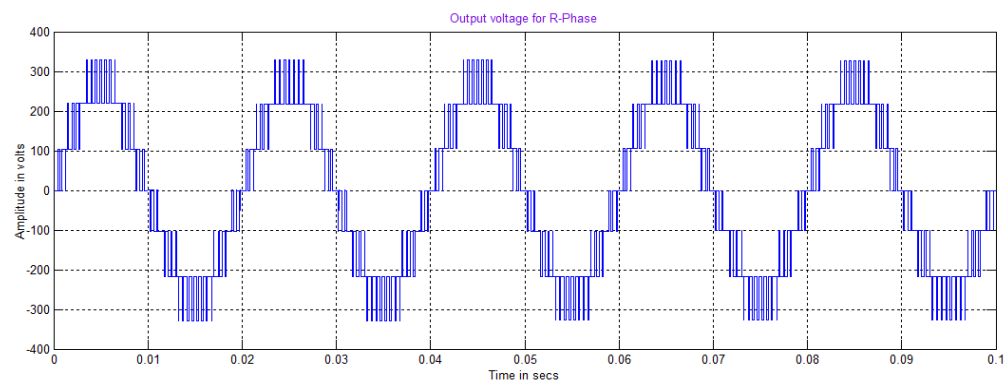

Figure 5. Total Output voltage of H-bridge + H-type FCMLI for R-Phase 


\section{PWM STRATEGIES}

High-switching-frequency modulation methods like multicarrier bipolar PWM and space vector modulation techniques have been used for MLI modulation control. The proposed topology can be modulated with any one of these methods with suitable adjustment. In the present work, the multicarrier bipolar PWM scheme is used. Figure 6 and 7 shows the sample carrier arrangement. In a multicarrier bipolar PWM scheme, carrier signals are compared with the reference signal, and the pulses obtained are used for switching of devices corresponding to respective voltage levels. In the proposed topology, one switch may contribute for synthesis of more than one level at output terminals.

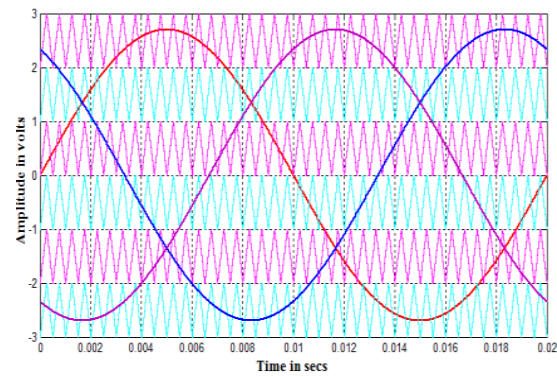

Figure 6. Sample carrier arrangement for sinusoidal reference and PDPWM strategy

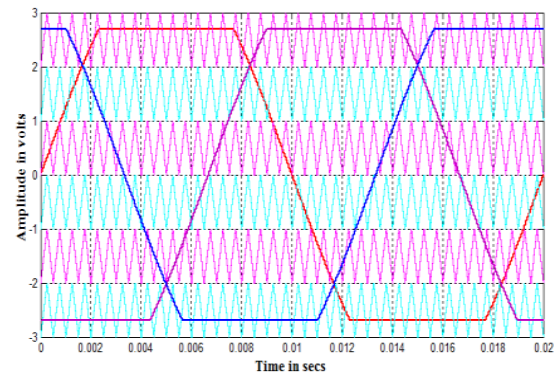

Figure 7. Sample carrier arrangement for 60 degree reference and PDPWM strategy

\section{SIMULATION RESULTS}

The simulated output voltage is shown for only one sample value of $\mathrm{m}_{\mathrm{a}}=0.8$. The following parameter values are used for simulation: $\mathrm{V}_{\mathrm{dc} 1}=110 \mathrm{~V}, \mathrm{~V}_{\mathrm{dc} 2}=440 \mathrm{~V}, \mathrm{R}(\mathrm{load})=100$ ohms, $\mathrm{C}_{1}=\mathrm{C}_{2}=10$ e- 3 Farad and $\mathrm{C}_{3}=1600$ Farad, $\mathrm{f}_{\mathrm{c}}=2000 \mathrm{~Hz}$ and $\mathrm{f}_{\mathrm{m}}=50 \mathrm{~Hz}$. Figure 8, 10, 12 and 14 shows the sample output voltage for PDPWM strategy with sinusoidal, THI, 60 degree and stepped wave reference and the corresponding FFT plot is shown in Figure 9, 11, 13 and 15. Table 2-5 show the comparison of \% THD, $\mathrm{V}_{\mathrm{RMS}}$ (fundamental), CF and FF for different PWM strategies with various references.

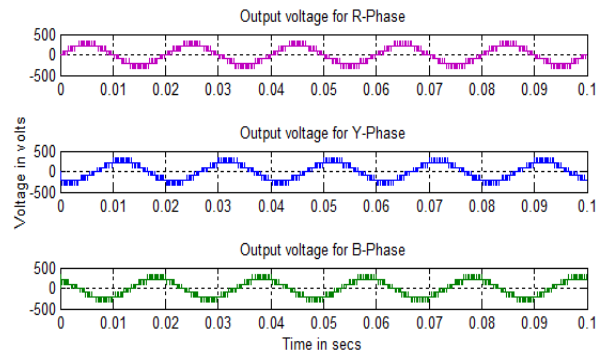

Figure 8. Sample output voltage generated by PDPWM strategy for H-bridge and H-type FCMLI with sine reference
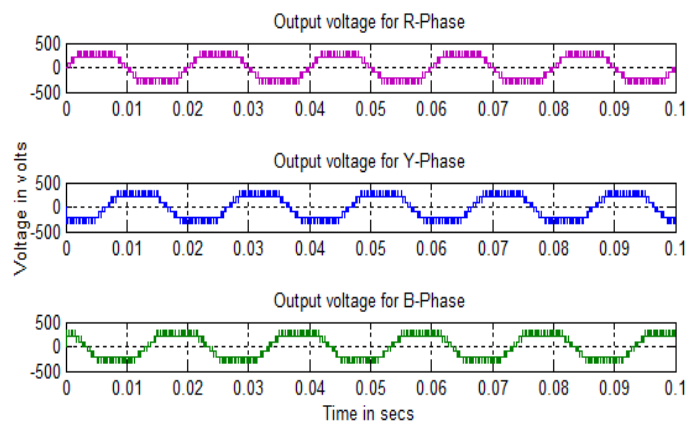

Figure 10. Sample output voltage generated by PDPWM strategy for H-bridge and H-type FCMLI with THI reference

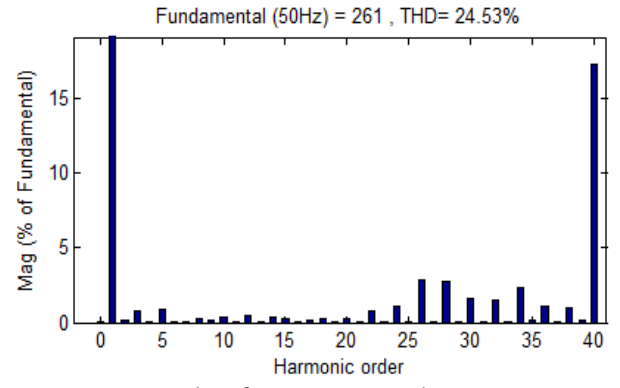

Figure 9. FFT plot for output voltage generated by PDPWM strategy for H-bridge and H-type FCMLI with sine reference

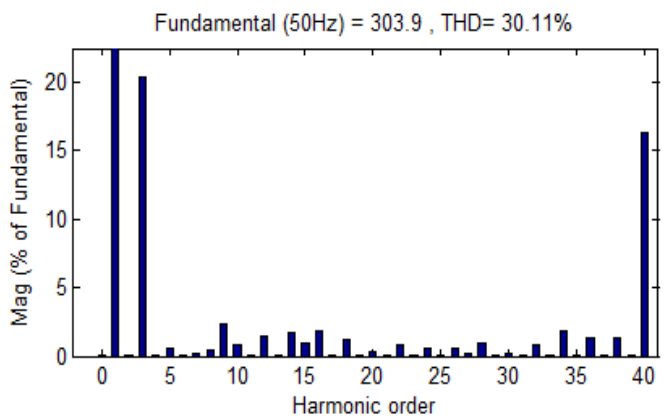

Figure 11. FFT plot for output voltage generated by PDPWM strategy for H-bridge and H-type FCMLI with THI reference 


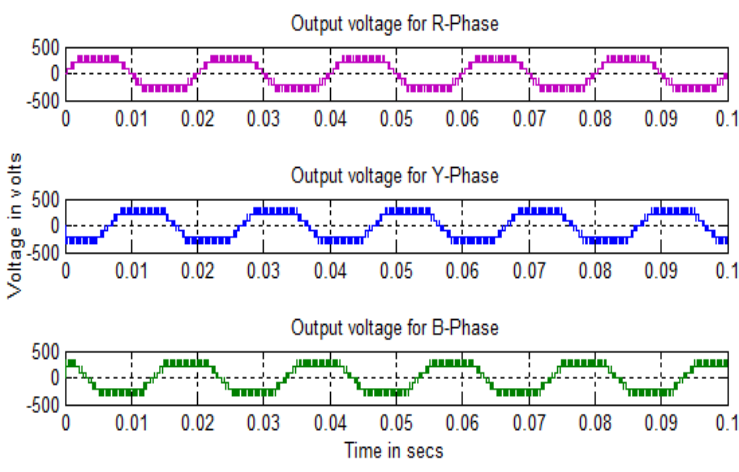

Figure 12. Sample output voltage generated by PDPWM strategy for H-bridge and H-type FCMLI with 60 degree reference

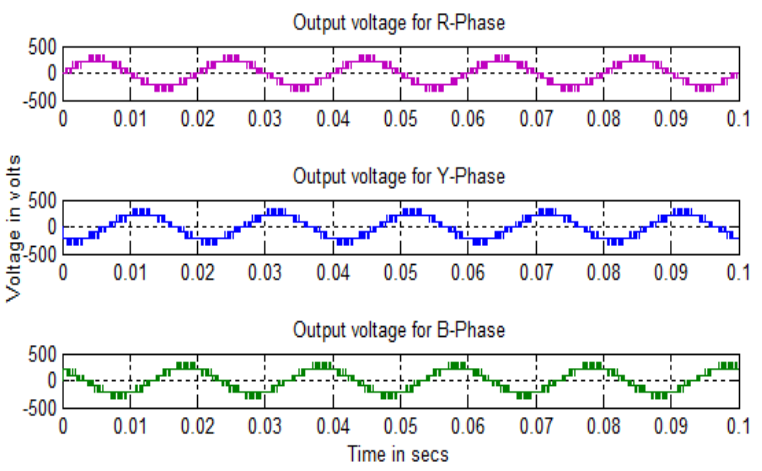

Figure 14. Sample output voltage generated by PDPWM strategy for H-bridge and H-type FCMLI with stepped wave reference

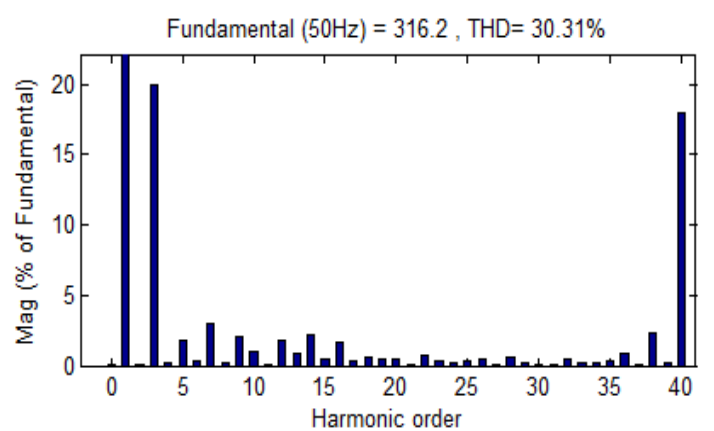

Figure 13. FFT plot for output voltage generated by PDPWM strategy for H-bridge and H-type FCMLI with 60 degree reference

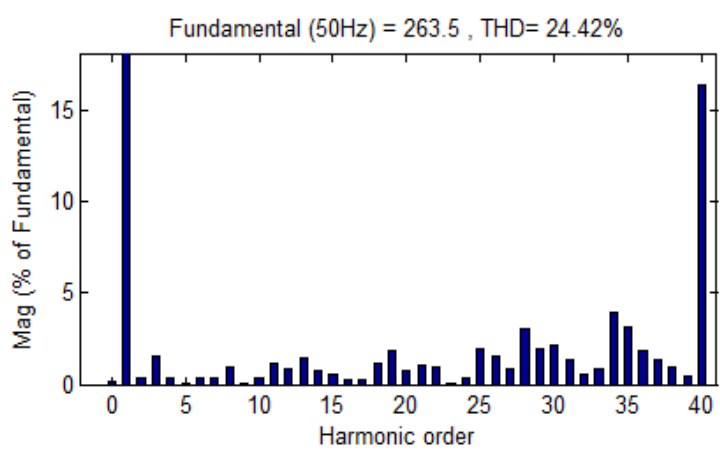

Figure 15. FFT plot for output voltage generated by PDPWM strategy for H-bridge and H-type FCMLI with stepped wave reference

Table 2. \% THD of output voltage (R-phase) of H- bridge and H-type FCMLI for various values of $\mathrm{m}_{\mathrm{a}}(\mathrm{R}-$ load, by simulation)

\begin{tabular}{|c|c|c|c|c|c|c|c|c|c|c|c|c|c|c|}
\hline \multirow[b]{2}{*}{$\mathrm{m}_{\mathrm{a}}$} & \multicolumn{7}{|c|}{ Sinusoidal reference } & \multicolumn{7}{|c|}{ Third harmonic injection reference } \\
\hline & PD & POD & APOD & $\mathrm{CO}-\mathrm{A}$ & $\mathrm{CO}-\mathrm{B}$ & $\mathrm{CO}-\mathrm{C}$ & VF & PD & POD & APOD & $\mathrm{CO}-\mathrm{A}$ & $\mathrm{CO}-\mathrm{B}$ & $\mathrm{CO}-\mathrm{C}$ & VF \\
\hline 1 & 18.35 & 18.28 & 18.11 & 22.81 & 21.26 & 16.85 & 18.41 & 24.97 & 25.06 & 24.92 & 28.22 & 28.04 & 26.37 & 24.98 \\
\hline 0.9 & 22.58 & 22.29 & 22.86 & 26.37 & 24.05 & 18.78 & 22.63 & 29.12 & 29.03 & 29.68 & 30.93 & 29.29 & 27.32 & 29.17 \\
\hline 0.8 & 24.53 & 24.32 & 24.39 & 29.38 & 27.83 & 21.90 & 24.56 & 30.11 & 29.92 & 30.92 & 32.84 & 31.46 & 29.47 & 30.16 \\
\hline 0.7 & 25.86 & 25.48 & 25.62 & 33.55 & 32.23 & 23.65 & 25.83 & 27.80 & 27.65 & 28.09 & 34.49 & 33.50 & 29.39 & 27.86 \\
\hline 0.6 & 34.75 & 34.66 & 34.06 & 40.98 & 40.68 & 25.21 & 34.62 & 36.66 & 36.14 & 35.72 & 41.86 & 42.28 & 29.28 & 36.71 \\
\hline \multirow{2}{*}{$\mathrm{m}_{\mathrm{a}}$} & \multicolumn{7}{|c|}{60 degree PWM reference } & \multicolumn{7}{|c|}{ Stepped wave reference } \\
\hline & $\mathrm{PD}$ & POD & APOD & $\mathrm{CO}-\mathrm{A}$ & $\mathrm{CO}-\mathrm{B}$ & $\mathrm{CO}-\mathrm{C}$ & VF & PD & POD & APOD & $\mathrm{CO}-\mathrm{A}$ & $\mathrm{CO}-\mathrm{B}$ & $\mathrm{CO}-\mathrm{C}$ & VF \\
\hline 1 & 22.69 & 22.49 & 22.59 & 26.88 & 28.10 & 26.47 & 22.72 & 16.78 & 16.87 & 17.12 & 22.39 & 22.64 & 17.84 & 16.77 \\
\hline 0.9 & 28.17 & 28.08 & 28.53 & 29.98 & 28.10 & 26.13 & 28.23 & 23.14 & 23.05 & 22.82 & 26.13 & 24.44 & 19.01 & 23.08 \\
\hline 0.8 & 30.31 & 29.94 & 31.26 & 32.44 & 30.94 & 29.16 & 30.39 & 24.42 & 24.69 & 23.92 & 29.10 & 27.70 & 20.48 & 24.41 \\
\hline 0.7 & 27.78 & 28.04 & 28.06 & 33.65 & 31.93 & 29.02 & 27.84 & 27.26 & 27.28 & 25.99 & 33.30 & 32.81 & 24.43 & 27.24 \\
\hline 0.6 & 34.05 & 33.79 & 33.53 & 40.62 & 40.47 & 29.98 & 34.06 & 34.52 & 35.27 & 32.91 & 40.98 & 40.41 & 27.04 & 34.53 \\
\hline
\end{tabular}

Table 3. $\mathrm{V}_{\mathrm{RMS}}$ of output voltage (R-phase) of $\mathrm{H}$ - bridge and H-type FCMLI for various values of $\mathrm{m}_{\mathrm{a}}\left(\mathrm{R}-\mathrm{load}_{\text {, }}\right.$ by simulation)

\begin{tabular}{|c|c|c|c|c|c|c|c|c|c|c|c|c|c|c|}
\hline \multirow[b]{2}{*}{$\mathrm{m}_{\mathrm{a}}$} & \multicolumn{7}{|c|}{ Sinusoidal reference } & \multicolumn{7}{|c|}{ Third harmonic injection reference } \\
\hline & PD & POD & APOD & $\mathrm{CO}-\mathrm{A}$ & $\mathrm{CO}-\mathrm{B}$ & $\mathrm{CO}-\mathrm{C}$ & VF & PD & POD & APOD & $\mathrm{CO}-\mathrm{A}$ & $\mathrm{CO}-\mathrm{B}$ & $\mathrm{CO}-\mathrm{C}$ & VF \\
\hline 1 & 231.8 & 231.5 & 232.6 & 247.1 & 255.5 & 255.2 & 231.8 & 268.7 & 269.5 & 268.8 & 276.6 & 283.3 & 282.9 & 268.7 \\
\hline 0.9 & 208.6 & 209.1 & 208.6 & 230.4 & 241.1 & 241 & 208.5 & 241.8 & 241.8 & 242.1 & 258.7 & 270.5 & 270.4 & 241.7 \\
\hline 0.8 & 184.5 & 184.5 & 185.6 & 212.4 & 222.6 & 222.3 & 184.6 & 214.9 & 214.8 & 215.5 & 240.8 & 252.8 & 252.7 & 214.8 \\
\hline 0.7 & 161 & 160.4 & 162.5 & 191.1 & 201.4 & 200.1 & 161 & 187.5 & 187.6 & 188.2 & 320.8 & 232.9 & 232.7 & 187.6 \\
\hline 0.6 & 136.7 & 136.7 & 138.8 & 164.2 & 173.1 & 172.1 & 136.7 & 159.6 & 159.7 & 161.4 & 189.1 & 199.9 & 199.4 & 159.6 \\
\hline
\end{tabular}




\begin{tabular}{|c|c|c|c|c|c|c|c|c|c|c|c|c|c|c|}
\hline \multirow[b]{2}{*}{$\mathrm{m}_{\mathrm{a}}$} & \multicolumn{7}{|c|}{60 degree PWM reference } & \multicolumn{7}{|c|}{ Stepped wave reference } \\
\hline & $\mathrm{PD}$ & POD & APOD & $\mathrm{CO}-\mathrm{A}$ & $\mathrm{CO}-\mathrm{B}$ & $\mathrm{CO}-\mathrm{C}$ & VF & PD & POD & APOD & $\mathrm{CO}-\mathrm{A}$ & CO-B & $\mathrm{CO}-\mathrm{C}$ & VF \\
\hline 0.9 & 251.6 & 251.8 & 252 & 265.8 & 277.4 & 277 & 251.6 & 209.8 & 210.1 & 212.5 & 230.5 & 242.7 & 240.7 & 209.8 \\
\hline 0.7 & 195.3 & 195.8 & 195.7 & 226.3 & 240.6 & 240.3 & 195.3 & 162 & 162.1 & 165.1 & 191.3 & 203.3 & 200.2 & 162 \\
\hline 0.6 & 166.3 & 166.1 & 167.6 & 197.1 & 208.1 & 207.4 & 166.3 & 137.3 & 136.8 & 140.1 & 165.8 & 173.2 & 171 & 137.2 \\
\hline
\end{tabular}

Table 4. CF of output voltage (R-phase) of $\mathrm{H}$ - bridge and H-type FCMLI for various values of $\mathrm{m}_{\mathrm{a}}(\mathrm{R}-\mathrm{load}$, by simulation)

\begin{tabular}{|c|c|c|c|c|c|c|c|c|c|c|c|c|c|c|}
\hline \multirow[b]{2}{*}{$\mathrm{m}_{\mathrm{a}}$} & \multicolumn{7}{|c|}{ Sinusoidal reference } & \multicolumn{7}{|c|}{ Third harmonic injection reference } \\
\hline & PD & POD & APOD & $\mathrm{CO}-\mathrm{A}$ & $\mathrm{CO}-\mathrm{B}$ & $\mathrm{CO}-\mathrm{C}$ & VF & PD & POD & APOD & $\mathrm{CO}-\mathrm{A}$ & $\mathrm{CO}-\mathrm{B}$ & $\mathrm{CO}-\mathrm{C}$ & VF \\
\hline 1 & 1.4141 & 1.4141 & 1.4140 & 1.4140 & 1.4144 & 1.4141 & 1.4141 & 1.4142 & 1.4144 & 1.4140 & 1.4143 & 1.4140 & 1.4142 & 1.4145 \\
\hline 0.9 & 1.4137 & 1.4139 & 1.4146 & 1.4144 & 1.4143 & 1.4141 & 1.4139 & 1.4139 & 1.4143 & 1.4142 & 1.4139 & 1.4144 & 1.4145 & 1.4145 \\
\hline 0.8 & 1.4146 & 1.4138 & 1.4137 & 1.4143 & 1.4141 & 1.4143 & 1.4138 & 1.4141 & 1.4143 & 1.4143 & 1.4140 & 1.4141 & 1.4143 & 1.4143 \\
\hline 0.7 & 1.4136 & 1.4142 & 1.4141 & 1.4139 & 1.4131 & 1.4142 & 1.4142 & 1.4144 & 1.4147 & 1.4144 & 1.4142 & 1.4139 & 1.4129 & 1.4141 \\
\hline 0.6 & 1.4147 & 1.4140 & 1.4135 & 1.4142 & 1.4142 & 1.4137 & 1.4140 & 1.4141 & 1.4139 & 1.4138 & 1.4145 & 1.4142 & 1.4132 & 1.4141 \\
\hline \multirow{2}{*}{$\mathrm{m}_{\mathrm{a}}$} & \multicolumn{7}{|c|}{60 degree PWM reference } & \multicolumn{7}{|c|}{ Stepped wave reference } \\
\hline & PD & POD & APOD & $\mathrm{CO}-\mathrm{A}$ & $\mathrm{CO}-\mathrm{B}$ & $\mathrm{CO}-\mathrm{C}$ & VF & PD & POD & APOD & $\mathrm{CO}-\mathrm{A}$ & $\mathrm{CO}-\mathrm{B}$ & $\mathrm{CO}-\mathrm{C}$ & VF \\
\hline 1 & 1.4138 & 1.4141 & 1.4142 & 1.4142 & 1.4140 & 1.4143 & 1.4142 & 1.4143 & 1.4145 & 1.4141 & 1.4145 & 1.4141 & 1.4143 & 1.4143 \\
\hline 0.9 & 1.4145 & 1.4142 & 1.4142 & 1.4142 & 1.4142 & 1.4144 & 1.4145 & 1.4142 & 1.4140 & 1.4141 & 1.4143 & 1.4140 & 1.4137 & 1.4142 \\
\hline 0.8 & 1.4141 & 1.4140 & 1.4152 & 1.4139 & 1.4141 & 1.4139 & 1.4141 & 1.4143 & 1.4146 & 1.4141 & 1.4142 & 1.4137 & 1.4142 & 1.4143 \\
\hline 0.7 & 1.4142 & 1.4141 & 1.4144 & 1.4144 & 1.4143 & 1.4140 & 1.4147 & 1.4141 & 1.4145 & 1.4136 & 1.4140 & 1.4146 & 1.4140 & 1.4135 \\
\hline 0.6 & 1.4143 & 1.4136 & 1.4146 & 1.4145 & 1.4142 & 1.4136 & 1.4137 & 1.4136 & 1.4144 & 1.4139 & 1.4143 & 1.4139 & 1.4146 & 1.4139 \\
\hline
\end{tabular}

Table 5. FF of output voltage (R-phase) of H- bridge and H-type FCMLI for various values of $\mathrm{m}_{\mathrm{a}}$ (R-load, by simulation)

\begin{tabular}{|c|c|c|c|c|c|c|c|c|c|c|c|c|c|c|}
\hline \multirow[b]{2}{*}{$\mathrm{m}_{\mathrm{a}}$} & \multicolumn{7}{|c|}{ Sinusoidal reference } & \multicolumn{7}{|c|}{ Third harmonic injection reference } \\
\hline & PD & POD & APOD & $\mathrm{CO}-\mathrm{A}$ & CO-B & $\mathrm{CO}-\mathrm{C}$ & VF & PD & POD & APOD & $\mathrm{CO}-\mathrm{A}$ & $\mathrm{CO}-\mathrm{B}$ & $\mathrm{CO}-\mathrm{C}$ & VF \\
\hline 1 & 1363.5 & 3311.4 & 7753.3 & 4118.3 & 12775 & 12760 & 3311.42 & 746.38 & 26950 & 26880 & 747.56 & 28330 & 28290 & 767.71 \\
\hline 0.9 & 1604.6 & 1603.8 & 5215 & 1645.7 & 12055 & 12050 & 1603.84 & 1209 & 12090 & 12105 & 3695.7 & 27050 & 27040 & 24170 \\
\hline 0.8 & 3890 & 1678.1 & 3712 & 21240 & 7420 & 7410 & 1678.18 & 7163.3 & 10740 & 10775 & 24080 & 25280 & 25270 & 7160 \\
\hline 0.7 & 847.36 & 670.83 & 2031.2 & 1124.1 & 5035 & 5002.5 & 670.83 & 3125 & 6253.3 & 6273.3 & 32080 & 11645 & 11635 & 1443.0 \\
\hline 0.6 & 3417.5 & 3417.5 & 1067.6 & 1642 & 2472.82 & 2458.57 & 3417.5 & 840 & 2281.4 & 2305.7 & 6303.3 & 49975 & 4985 & INF \\
\hline \multirow[b]{2}{*}{$\mathrm{m}_{\mathrm{a}}$} & \multicolumn{7}{|c|}{60 degree PWM reference } & \multicolumn{7}{|c|}{ Stepped wave reference } \\
\hline & PD & POD & APOD & $\mathrm{CO}-\mathrm{A}$ & CO-B & $\mathrm{CO}-\mathrm{C}$ & VF & PD & POD & APOD & $\mathrm{CO}-\mathrm{A}$ & $\mathrm{CO}-\mathrm{B}$ & $\mathrm{CO}-\mathrm{C}$ & VF \\
\hline 1 & 2543.6 & 28010 & 28000 & 944.66 & 28550 & 28500 & 2798 & 9336 & 7800 & 234.7 & 3108.7 & 12785 & 12780 & 416.78 \\
\hline 0.9 & 12580 & 25180 & 25200 & 984.44 & 27740 & 27700 & 6290 & 999.04 & 5252.5 & 212.5 & 794.82 & 12135 & 8023.3 & 428.16 \\
\hline 0.8 & 2795 & 11170 & 11195 & 24640 & 25740 & 25750 & 1490.66 & 1552.5 & 2315.7 & 189.3 & 646.66 & 7460 & 7363.3 & 503.51 \\
\hline 0.7 & 1395 & 6526.6 & 6523.3 & 2514.4 & 12030 & 12015 & 781.2 & 5400 & 1140 & 165.1 & 1125.2 & 5082.5 & 5005 & 540 \\
\hline 0.6 & 475.14 & 2768.3 & 2793.3 & 19710 & 6936.66 & 6913.3 & 1847.77 & 624.09 & 3724 & 140.1 & 1658 & 2886.6 & 2442.8 & 4573.3 \\
\hline
\end{tabular}

\section{CONCLUSION}

In this paper, a three phase new hybrid topology of H-bridge $+\mathrm{H}$-type FCMLI using sinusoidal reference, third harmonic injection reference, 60 degree reference and stepped wave reference are initially developed using SIMULINK. The five level output voltages of the chosen MLIs obtained using the MATLAB based PWM strategies and the corresponding \% THD, $\mathrm{V}_{\mathrm{RMS}}$ (fundamental), CF and FF are presented and analyzed. From tables it is observed that bipolar COPWM-C provides output with relatively low distortion for sine reference and bipolar COPWM-B strategy is found to perform better since it provides relatively higher fundamental RMS output voltage for 60 degree reference.

\section{REFERENCES}

[1] E.Babaei, et al., "A New General Topology for Cascaded Multilevel Inverters With Reduced Number of Components Based on Developed H-Bridge", IEEE Trans. Ind. Electron, Vol. 61, No. 8, pp. 3932-3939, 2014.

[2] M. R. Banaei, et al., "Reconfiguration of semi-cascaded multilevel inverter to improve systems performance parameters”, IET Power Electron, Vol. 7, No. 5, pp. 1106-1112, 2014.

[3] M. F. Kangarlu and E. Babaei, "A Generalized Cascaded Multilevel Inverter Using Series Connection of Submultilevel Inverters", IEEE Tran. Power Electron, Vol. 28, No. 2, pp. 625-636, 2013.

[4] C. I. Odeh and D. B. N. Nnadi, "Single-phase 9-level hybridised cascaded multilevel inverter", IET. Power Electron, Vol. 6, No. 3, pp. 468-477, 2013.

[5] Chung-Ming Young, et al., "A Single-Phase Multilevel Inverter with Battery Balancing”, IEEE Tran. Ind. Electron, Vol. 60, No. 5, pp. 1972-1978, 2013. 
[6] A. K. Panda and Y. Suresh, "Performance of cascaded multilevel inverter by employing single and three-phase transformers", IET Power Eletron, Vol. 5, No. 9, pp. 1694-1705, 2012.

[7] Li Zixin, et al., "A Novel Single-Phase Five-Level Inverter With Coupled Inductors", IEEE Trans. Power Electron, Vol. 27, No. 6, pp. 2716-2725, 2012.

[8] Yi-Hung Liao and Ching-Ming Lai, "Newly-Constructed Simplified Single-Phase Multistring Multilevel Inverter Topology for Distributed Energy Resources", IEEE Trans. Power Electron, Vol. 26, No. 9, pp. 2386-2392, 2011.

[9] Y. Hinago and H. Koizumi, "A Single-Phase Multilevel Inverter Using Switched Series/Parallel DC Voltage Sources", IEEE Trans. Ind. Electron, Vol. 57, No. 8, pp. 2643-2650, 2010.

[10] V. Naumanen, et al, "Mitigation of high du/dt-originated motor overvoltages in multilevel inverter drives", IET Power Electron, Vol. 3, No. 5, pp. 681-689, 2010.

\section{BIOGRAPHIES OF AUTHORS}

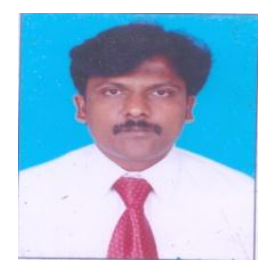

C.R.Balamurugan was born in 1978 in Kumbakonam. He has obtained B.E (Electrical and Electronics) and M.E (Power Electronics and Drives) degrees in 2000 and 2005 respectively from Arunai Engineering College, Tiruvannamalai and Sathyabama University, Chennai. He has been working in the teaching field for about 10 years. His areas of interest include power electronics, electrical machines and solar energy systems. He has 30 publications in international journals. His research papers 25 have been presented in various/IEEE international/national conferences. Currently, he is working as Assistant Professor in the Department of EEE, Arunai Engineering College, Tiruvannamalai. He is a life member of Instrument Society of India and Indian Society for Technical Education. Contact number- +91-9894522351. Email:crbalain2010@gmail.com.

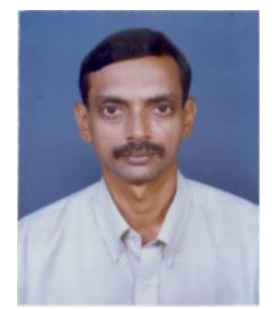

Dr. S.P.Natarajan was born in 1955 in Chidambaram. He has obtained B.E (Electrical and Electronics) and M.E (Power Systems) degrees in 1978 and 1984 respectively from Annamalai University securing distinction and then Ph.D in Power Electronics from Anna University, Chennai in 2003. He is currently Professor and Head of Instrumentation Engineering Department at Annamalai University where he has put in 31 years of service. He produced eight Ph.Ds and presently guiding eight Ph.D Scholars and so far guided eighty M.E students. His research papers 66 have been presented in various/IEEE international/national conferences in Mexico, Virginia, Hong Kong, Malaysia, India, Singapore and Korea. He has 20 publications in national journals and 43 in international journals. His research interests are in modeling and control of DC-DC converters and multiple connected power electronic converters, control of permanent magnet brushless DC motor, embedded control for multilevel inverters and matrix converters etc. He is a life member of Instrument Society of India and Indian Society for Technical Education. Contact number- +91-9443185211. Email: spn_annamalai @ rediffmail.com.

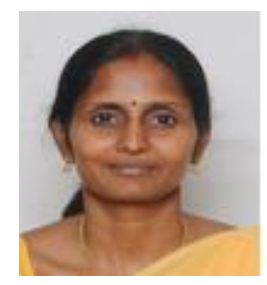

Dr. V.Padmathilagam was born in 1972 in Cuddalore. She has obtained B.E (Electrical and Electronics Engineering), M.E (Power Systems) and Ph.D from Annamalai University, Chidambaram. She is presently a Assistant Professor in EEE of Annamalai University where she has put in a total service of 14 years. Her research papers (04) have been presented in various / IEEE international /national conferences. She has 5 publications in national journal and 14 in international journals. Her areas of interest are: Power Electronics, Electronics, Neural and Fuzzy networks, Power Quality. Contact number- +91-9443929800. Email: vpt_au@yahoo.co.in.

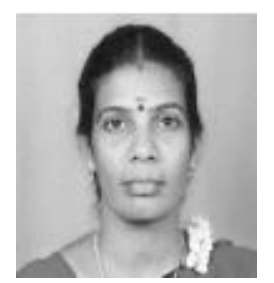

Dr. T.S.Anandhi obtained the B.E. (Electronics and Instrumentation), M.E (Process Control and Instrumentation) and Ph.D degrees from Annamalai University She is presently a Reader in the Department of Instrumentation Engineering, Annamalai University where she has put in a total service of 16 years. Her research interests are in modeling and control of multiple connected DC-DC converters and multilevel inverters. 